\title{
Position-Based Compressed Channel Estimation and Pilot Design for High-Mobility OFDM Systems
}

\author{
Xiang Ren, Wen Chen, Senior Member, IEEE, and Meixia Tao, Senior Member, IEEE
}

\begin{abstract}
With the development of high-speed trains (HSTs) in many countries, providing broadband wireless services in HSTs is crucial. Orthogonal frequency-division multiplexing (OFDM) has been widely adopted for broadband wireless communications due to its high spectral efficiency. However, OFDM is sensitive to the time selectivity caused by high-mobility channels, which costs much spectrum or time resources to obtain the accurate channel state information (CSI). Therefore, the channel estimation in high-mobility OFDM systems has been a long-standing challenge. In this paper, we first propose a new position-based high-mobility channel model, in which the HST's position information and Doppler shift are utilized to determine the positions of the dominant channel coefficients. Then, we propose a joint pilot placement and pilot symbol design algorithm for compressed channel estimation. It aims to reduce the coherence between the pilot signal and the proposed channel model and, hence, can improve channel estimation accuracy. Simulation results demonstrate that the proposed method performs better than existing channel estimation methods over high-mobility channels. Furthermore, we give an example of the designed pilot codebook to show the practical applicability of the proposed scheme.
\end{abstract}

Index Terms-Channel estimation, compressed sensing (CS), high-mobility channels, orthogonal frequency-division multiplexing (OFDM), position-based channel model.

\section{INTRODUCTION}

$\mathbf{O}$ RTHOGONAL frequency-division multiplexing (OFDM) has been widely adopted for broadband wireless communication systems due to its high spectral efficiency [1]. In OFDM systems, each subcarrier has a narrow bandwidth that ensures that the signal is robust against the frequency selectivity caused by the multipath delay spread. However, OFDM is sensitive to the time selectivity, which is induced by rapid time variations of mobile channels. In recent years, high-speed trains (HSTs) have been increasingly developed in many countries,

Manuscript received April 18, 2014; accepted July 4, 2014. Date of publication July 22, 2014; date of current version May 12, 2015. This work was supported in part by the National 973 Project under Grant 2012CB316106; by the National Natural Science Foundation of China under Grant 61161130529, Grant 61328101, and Grant 61322102; by the Science and Technology Commission of Shanghai Municipality through the Science and Technology Innovation Program under Grant 13510711200; and by the Southeast University National Key Laboratory on Mobile Communications under Grant 2013D11. The review of this paper was coordinated by Prof. S. Muhaidat.

$\mathrm{X}$. Ren and M. Tao are with the Department of Electronic Engineering, Shanghai Jiao Tong University, Shanghai 200240, China (e-mail: renx@ sjtu. edu.cn; mxtao@sjtu.edu.cn).

W. Chen is with the Department of Electronic Engineering, Shanghai Jiao Tong University, Shanghai 200240, China, and also with the School of Electronic Engineering and Automation, Guilin University of Electronic Technology, Guilin 541004, China (e-mail: wenchen@ sjtu.edu.cn).

Color versions of one or more of the figures in this paper are available online at http://ieeexplore.iee.org.

Digital Object Identifier 10.1109/TVT.2014.2341712 and OFDM has been adopted for high-data-rate services [2]. Since an HST travels at a speed of around $500 \mathrm{~km} / \mathrm{h}$, the wireless channels suffer from a high Doppler shift. In highmobility environments, wireless channels are both fast timevarying and frequency selective and can be considered the doubly selective channels [3]-[5]. As the quality of channel estimation has a major impact on the overall system performance, it is necessary to investigate reliable estimation methods in high-mobility environments.

Channel estimation for fast time-varying channels has been extensively studied in the literature, and various time-varying channel models have been established. Sung and Brady[6] and Mostofi and Cox [7] proposed several channel estimators by using a linear time-varying channel model. They assumed that the channel varies with time linearly in one or more OFDM symbols. The method in [6] works well at low Doppler shifts since some channel matrix coefficients are ignored. Mostofi and Cox [7] proposed two approaches to estimate time-varying channels: one using guard intervals and the other exploiting three consecutive symbols. However, the linear models can result in large modeling error and severely degrade the channel estimation performance in high-mobility environments, where the channel may change significantly even within one OFDM symbol. To overcome the modeling problem, in [8]-[10], several basis expansion models (BEMs) were proposed for the time variations of each OFDM symbol. Hijazi and Ros [8] assumed a polynomial BEM channel model and suggested an iterative channel estimation method. Tang et al. [9] proposed channel estimators based on a windowed BEM to combat both the noise and the out-of-band interference. In particular, Ma et al. [10] claimed that the equidistant pulse-shaped pilot placement is optimal for a BEM-based doubly selective channel. These works are based on the implicit assumption of rich underlying multipath environments.

Recently, growing experimental studies have shown that the high-mobility channels tend to exhibit a sparse structure at some high-dimensional signal spaces, such as the delayDoppler domain, and can be characterized by significantly fewer parameters. To utilize the inherent channel sparsity, in [3]-[5], [11], and [12], the applications of compressed sensing (CS) in doubly-selective channels, which well reflect the nature of the high-mobility channels, were studied. In [3]-[5], the notion of channel sparsity was introduced and CS-based approaches were presented to estimate the channel state information (CSI). In [11] and [12], the delay-Doppler basis was optimized to improve the estimation performances.

Coherence is an important issue in CS, and fundamental studies [13]-[16] show that the coherence influences the CS 


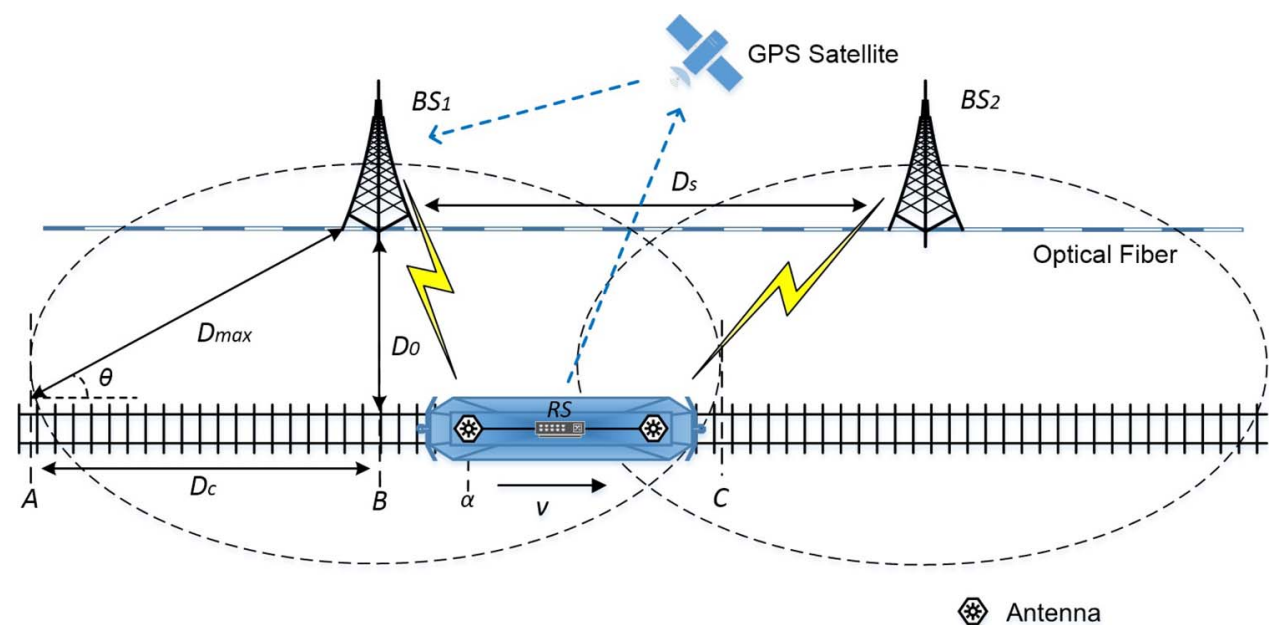

Fig. 1. Structure of the HST communication system.

reconstruction performance directly. In [13]-[15], it was concluded that lower coherence between the measurement matrix and the dictionary matrix in CS leads to better performance. Therefore, how to the pilot signal to reduce the system coherence in a high-mobility environment is a very interesting and valuable problem. In [17]-[23], several pilot design methods to minimize the CS coherence and improve the system performance were studied. In [17]-[19], several pilot placement design methods based on an exhaustive search to reduce the system coherence in sparse channels with large iterations were proposed. In [20] and [21], the pilot placement was designed by using a discrete stochastic approximation method for sparse OFDM channels without considering the channel mobility and the intercarrier interference (ICI). In an earlier work [22], we designed the pilot symbol by shrinking the Gram matrix in a high-mobility multiple-input-multiple-output (MIMO) OFDM system. However, none of these works considered the joint optimization of the pilot symbol and pilot placement.

In this paper, we introduce a new position-based compressed channel estimation method for OFDM systems over highmobility channels, in which the pilot placement and pilot symbol are jointly designed to further improve the performance. In specific, we propose a new position-based high-mobility channel model that reflects the Doppler shift according to the HST position. The HST's position information and Doppler shift are utilized to predict the positions of the sparse dominant channel coefficients, which highly reduces the estimation complexity. Then, based on the CS coherence minimization criterion, a joint pilot placement and pilot symbol design algorithm is proposed to reduce the coherence between the pilot signal and the proposed channel model. Simulation results demonstrate that the proposed method achieves better performances than existing channel estimation methods in the high-mobility environment. Furthermore, we give an example of a designed pilot codebook to show the practical applicability of the proposed scheme.

The remainder of this paper is organized as follows. Section II introduces the HST communication model, the OFDM system model, and the conventional high-mobility channel model. In Section III, a new position-based highmobility channel model is proposed. Then, we introduce a new position-based channel estimation method and discuss the ICI mitigation method. In Section IV, we propose a joint pilot placement and pilot symbol design algorithm and discuss its complexity and convergence. The practical applicability of the proposed scheme is also discussed. Section V presents simulation results in the high-mobility environment. Finally, Section VI concludes this paper.

Notations: $\|\cdot\|_{\ell_{0}}$ denotes the number of nonzero entries in a matrix or vector, and $\|\cdot\|_{\ell_{2}}$ is the Euclidean norm. Notation $\phi(k, u)$ denotes the $(k, u)$ th entry of the matrix $\boldsymbol{\Phi}$, and $\phi(:, u)$ denotes the $u$ th column vector of the matrix $\boldsymbol{\Phi}$. The superscripts $(\cdot)^{T}$ and $(\cdot)^{H}$ denote the transposition and Hermitian of a matrix, respectively. $\otimes$ denotes the Kronecker product, and $\mathbf{a}=$ vec $\{\mathbf{A}\}$ denotes the vector obtained by stacking the columns of matrix A. Finally, $\mathbb{R}$ denotes the real field, and $\mathbb{Z}$ denotes the set of integers.

\section{SYSTEM MODEL}

\section{A. High-Speed Train Communications}

We consider a well-recognized system architecture of broadband wireless communications for HSTs [2], [24], as shown in Fig. 1. The communication between base stations (BSs) and mobile users is conducted in a two-hop manner through a relay station (RS) deployed on the train. The RS has two antennas on the top of the train to communicate with the BS. On the other hand, multiple indoor antennas are distributed in the train carriages to communicate with mobile users by existing wireless communication technologies, e.g., wireless fidelity (Wi-Fi). The BSs are located $10-50 \mathrm{~m}$ away from the railway at some intervals and connected with optical fibers. Here, we assume each BS is equipped with one antenna and has the same power allocation and coverage range.

In this paper, we focus on the channel estimation between the BSs and the RS on the HST. When the HST camps in a single cell, the RS selects the antenna with better channel quality to communicate with the BS; when the HST moves across the cell edges, the front antenna executes handover, whereas the rear one remains connected to the serving BS. The HST is equipped 
with a GPS, which can estimate the HST's instant position and speed information and send them to the BS [25]. Several factors may influence the performance of the GPS, such as signal arrival time measurement, atmospheric effects, terrains, and so on. In particular, when the HST runs in a tunnel, the GPS accuracy may be highly reduced. However, in this paper, we do not consider these factors and assume that the HST runs in a plain terrain. We also assume that the GPS estimates the HST's speed and position information perfectly and sends the information to the BS with no time delay.

Denote $v$ as the speed of the HST and $c$ as the light speed. The distance between BSs is denoted as $D_{s}$. Let $D_{\max }$ denote the maximum distance of the coverage of the BS to the railway, i.e., positions $A$ and $C$ to $\mathrm{BS}_{1}$. Let $D_{0}$ denote the minimum distance, i.e., the position $B$ to $\mathrm{BS}_{1}$, and $D_{c}$ denote the distance between $A$ and $B$. In each cell, we define the HST position $\alpha$ as the distance between the serving antenna and the position $A$, and $\alpha=0$ at $A$. Let $\theta$ denote the angle between the signal transmitted from the BS to RS and the railway. When the HST moves from $A$ to $C, \theta$ changes from $\theta_{\min }$ to $\theta_{\max }$. If $D_{\max } \gg$ $D_{0}$, then we have $\theta_{\min } \approx 0^{\circ}$ and $\theta_{\max } \approx 180^{\circ}$. Furthermore, HST suffers from the Doppler shift $f_{d}$ at different positions, and $f_{d}$ can be calculated by using the equation $f_{d}=(v / c)$. $f_{c} \cos \theta$, in which $f_{c}$ is the carrier frequency. It is easy to find that $f_{d_{0}}=0$ at $B$ for $\theta=90^{\circ}$.

\section{B. OFDM System}

We consider an OFDM system with $K$ subcarriers for the link between the BS and the RS in the HST communication system. The transmit signal at the $k$ th subcarrier of the $n$th OFDM symbol is denoted as $X^{n}(k)$, for $n=1,2, \ldots, N$ and $k=1,2, \ldots, K$. The transmitter performs the inverse discrete Fourier transform (DFT), inserts the cyclic prefix (CP), and then transmits the signals to the channel. After removing the $\mathrm{CP}$ and passing the DFT operation at the receiver, the received signal in the frequency domain can be represented as

$$
\mathbf{Y}^{n}=\mathbf{H}^{n} \mathbf{X}^{n}+\mathbf{W}^{n}
$$

where $\mathbf{Y}^{n}=\left[Y^{n}(1), Y^{n}(2), \ldots, Y^{n}(K)\right]^{T}$ is the received signal vector over all subcarriers during the $n$th OFDM symbol, $\mathbf{H}^{n}$ is a $K \times K$ channel matrix in the frequency domain, $\mathbf{X}^{n}=\left[X^{n}(1), X^{n}(2), \ldots, X^{n}(K)\right]^{T}$ is the transmitted signal vector over all subcarriers, and $\mathbf{W}^{n}=\left[W^{n}(1), W^{n}(2), \ldots\right.$, $\left.W^{n}(K)\right]^{T}$ denotes the noise vector, where $W^{n}(k)$ is the additive white Gaussian noise (AWGN) with a zero mean and $\sigma_{W}^{2}$ variance. The entries of $\mathbf{H}^{n}$ are represented as

$$
\begin{array}{r}
H^{n}(k, d)=\frac{1}{K} \sum_{m=0}^{K-1} \sum_{\ell=0}^{I-1} h^{n}(\ell, m) e^{-j \frac{2 \pi}{K} \ell(k-1)} e^{j \frac{2 \pi}{K}(d-k) m} \\
1 \leq k ; \quad d \leq K
\end{array}
$$

where $h^{n}(\ell, m)$ is the $\ell$ th channel tap in the $m$ th sample time of the $n$th OFDM symbol, and $I$ is the maximum number of channel taps. More detailed descriptions of $\mathbf{H}^{n}$ in highmobility environments is given in the following.
If the channel is time invariant, the off-diagonal term $H^{n}(k, d) \quad(k \neq d)$ is negligible, and the diagonal term $H^{n}(k, d)(k=d)$ alone represents the channel in the frequency domain. Therefore, channel matrix $\mathbf{H}^{n}$ can be divided into two parts as the ICI-free channel matrix $\mathbf{H}_{\text {free }}^{n} \triangleq \operatorname{diag}\left\{\left[H^{n}(1,1)\right.\right.$, $\left.\left.H^{n}(2,2), \ldots, H^{n}(K, K)\right]\right\}$ and the ICI channel matrix $\mathbf{H}_{\mathrm{ICI}}^{n} \triangleq$ $\mathbf{H}^{n}-\mathbf{H}_{\text {free }}^{n}$. Then, (1) can be rewritten as

$$
\begin{aligned}
\mathbf{Y}^{n} & =\mathbf{H}_{\text {free }}^{n} \mathbf{X}^{n}+\mathbf{H}_{\mathrm{ICI}}^{n} \mathbf{X}^{n}+\mathbf{W}^{n} \\
& =\mathbf{X}_{d}^{n} \mathbf{H}_{v e c}^{n}+\mathbf{H}_{\mathrm{ICI}}^{n} \mathbf{X}^{n}+\mathbf{W}^{n}
\end{aligned}
$$

where $\mathbf{X}_{d}^{n}=\operatorname{diag}\left\{\left[X^{n}(1), X^{n}(2), \ldots, X^{n}(K)\right]^{T}\right\}$ is a diagonal matrix of $\mathbf{X}^{n}$, and $\mathbf{H}_{v e c}^{n}=\operatorname{vec}\left\{\mathbf{H}_{\text {free }}^{n}\right\}$ is the stacking vector of $\mathbf{H}_{\text {free. }}^{n}$.

\section{High-Mobility Channel Model}

Let $\tau_{\max }$ be the maximum delay spread, $f_{d_{\max }}$ be the maximum Doppler shift, $T_{d}$ be the packet duration, and $W$ be the system bandwidth. Denote $T_{0}$ as the OFDM symbol duration and $W_{0}$ as the bandwidth of each subcarrier, $N_{t}=T_{d} / T_{0}$ and $N_{f}=W / W_{0}$. The high-mobility channel at the $k$ th subcarrier of the $n$th OFDM symbol in the delay-Doppler domain [3]-[5] can be modeled as

$$
H(n, k)=\sum_{l=0}^{L-1} \sum_{m=-M}^{M} \beta_{l, m} e^{j 2 \pi \frac{m}{N_{t}} n} e^{-j 2 \pi \frac{l}{N_{f}} k}
$$

where $L=\left\lceil W \tau_{\max }\right\rceil+1$ represents the maximum number of resolvable paths, $M=\left\lceil 2 T_{d} f_{d_{\max }}\right\rceil$ represents the maximum number of resolvable Doppler shifts, and $\beta_{l, m}$ is the channel coefficient of the $l$ th resolvable path with the resolvable Doppler shift $m$. For convenience, we define two vectors $\mathbf{u}_{k}=$ $\left[1, e^{-j 2 \pi\left(1 / N_{f}\right) k}, \ldots, e^{-j 2 \pi\left((L-1) / N_{f}\right) k}\right]$ and $\mathbf{u}_{n}=\left[e^{j 2 \pi\left(-M / N_{t}\right) n}\right.$, $\left.\left.e^{j 2 \pi\left((-M+1) / N_{t} n\right.}\right), \ldots, e^{j 2 \pi\left(M / N_{t}\right) n}\right]$. Then, the channel model can be represented as a matrix form:

$$
H(n, k)=\mathbf{u}_{k} \mathbf{B} \mathbf{u}_{n}^{T}=\left(\mathbf{u}_{n} \otimes \mathbf{u}_{k}\right) \mathbf{b}
$$

where $\mathbf{B}$ is an $L \times(2 M+1)$ channel coefficient matrix in the delay-Doppler domain, i.e.,

$$
\mathbf{B} \triangleq\left[\begin{array}{ccccc}
\beta_{0,-M} & \cdots & \beta_{0,0} & \cdots & \beta_{0, M} \\
\vdots & \ddots & \vdots & \ddots & \vdots \\
\beta_{L-1,-M} & \cdots & \beta_{L-1,0} & \cdots & \beta_{L-1, M}
\end{array}\right]
$$

and $\mathbf{b} \triangleq \operatorname{vec}\{\mathbf{B}\}$ is the stacking vector of $\mathbf{B}$, i.e.,

$$
\begin{aligned}
\mathbf{b} & =\left[\mathbf{b}_{-M}^{T}, \ldots, \mathbf{b}_{0}^{T}, \ldots, \mathbf{b}_{M}^{T}\right]^{T} \\
& =\left[\beta_{0,-M}, \ldots, \beta_{L-1,-M}, \ldots, \beta_{0, M}, \ldots, \beta_{L-1, M}\right]^{T}
\end{aligned}
$$

where $\mathbf{b}_{x}$ denotes the column vector of $\mathbf{B}$, and $x=-M$, $-M+1, \ldots, M$.

To explore the sparsity of the high-mobility channel, here, we introduce the definition of $S$-sparse channel based on [4]. In general, the high-mobility channel is $S$-sparse in the delayDoppler domain, due to the large number of the nondominant 


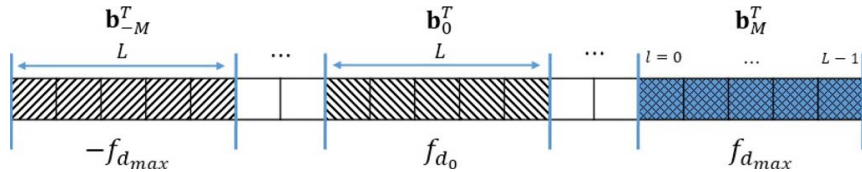

Fig. 2. Structure of the coefficient vector $\mathbf{b}^{T}$.

channel coefficients [5]. In this paper, we assume that the coefficients are constant within each OFDM symbol and different for different symbols.

Definition 1 (S-sparse Channels [4]): Define the dominant coefficients of a wireless channel as the coefficients that contribute significant power, i.e., $\left|\beta_{l, m}\right|^{2}>\gamma$, where $\gamma$ is a chosen threshold. We say that the channel is $S$-sparse if the number of its dominant coefficients satisfies $S=\|\mathbf{b}\|_{\ell_{0}} \ll$ $N_{0}=L(2 M+1)$, where $N_{0}$ is the total number of the channel coefficients.

\section{Position-BASEd High-Mobility CHANNEL ESTIMATION}

Here, we first propose a new position-based channel model to reduce the number of channel estimation parameters by utilizing the position information. Then, based on the proposed channel model, we give a position-based channel estimation scheme. Finally, the ICI mitigation scheme is also discussed.

\section{A. Position-Based High-Mobility Channel Model}

Considering the HST communication system model shown in Fig. 1. When the HST is at a certain position $\alpha$ with speed $v$, the high-mobility channel suffers from a certain Doppler shift $f_{d}$. Here, we assume that $v$ is constant during the HST passing a cell, and $f_{d}$ is constant in one OFDM symbol. In this case, it can be found that the dominant coefficients only exist in the $\mathbf{b}_{x}$ for suffering the same $f_{d}$, which can be represented as

$$
\mathbf{b}_{x}=\left[\beta_{0, x}, \beta_{1, x}, \ldots, \beta_{L-1, x}\right]^{T} .
$$

This is reasonable since $\mathbf{b}_{x}$ represents the $L$ resolvable paths with the resolvable Doppler shift $m$. The relationship between the subscript $x$ and $f_{d}$ is given as

$$
x= \begin{cases}\left\lceil 2 T_{d} f_{d}\right\rceil, & f_{d} \in\left[0, f_{d_{\max }}\right] \\ \left\lfloor 2 T_{d} f_{d}\right\rfloor, & f_{d} \in\left[-f_{d_{\max }}, 0\right) .\end{cases}
$$

Denote $\tilde{M}=2 T_{d} f_{d_{\max }}=2 T_{d}(v / c) \cdot f_{c}$. Then, the relationship between $x$ and $\alpha$ can be represented as

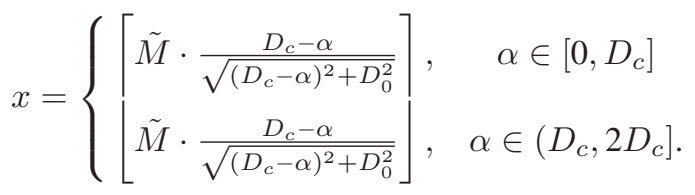

Note that, when $D_{\max } \gg D_{0}$, we have $D_{c}=D_{\max }$.

The structure of the coefficient vector $\mathbf{b}^{T}$ is shown as Fig. 2. In particular, $\mathbf{b}_{M}^{T}(x=M)$ with the cross lines denotes the column vector including the dominant coefficients with $f_{d_{\max }}$ at the position $A, \mathbf{b}_{0}^{T}(x=0)$ with the slash lines denotes the

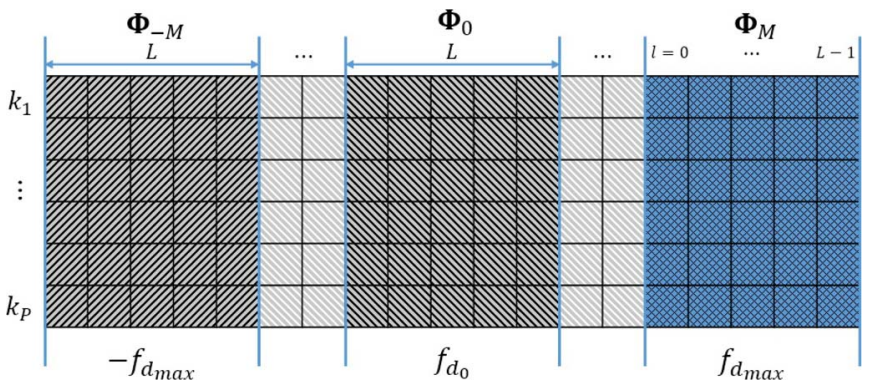

Fig. 3. Structure of the high-mobility channel model matrix $\mathbf{\Phi}$.

one with $f_{d_{0}}=0$ at $B$, and $\mathbf{b}_{-M}^{T}(x=-M)$ with the back slash lines denotes the one with $-f_{d_{\max }}$ at $C$, respectively. When the HST moves from $A$ to $C, \mathbf{b}_{x}^{T}$ changes from $\mathbf{b}_{M}^{T}$ to $\mathbf{b}_{-M}^{T}$ in sequence. On the other hand, other coefficients are nondominant, which contribute less to the CSI, according to Definition 1.

Remark: (Channel Sparsity): It is easy to find that, with a certain $f_{d}, \mathbf{b}_{x}$ contains at most $L$ dominant coefficients, and the sparsity is $S$, i.e., $\left\|\mathbf{b}_{x}\right\|_{\ell_{0}}=\|\mathbf{b}\|_{\ell_{0}}=S \leq L<L(2 M+1)$. Furthermore, high-mobility channels are considered the doubly selective channels in [3]-[5] and have the multipath sparsity, which means there are only $S$ paths $(S \ll L)$ with large coefficients and others can be neglected. In addition, as $M$ increases with the Doppler shift caused by the HST speed, high mobility will introduce a large $M$. Therefore, we have $\left\|\mathbf{b}_{x}\right\|_{\ell_{0}}=S \ll$ $L \ll L(2 M+1)$, and the high-mobility channel is $S$-sparse in the proposed position-based model.

After knowing the position of $\mathbf{b}_{x}$, which includes the dominant coefficients, we can further get the dominant channel model $\boldsymbol{\Phi}_{x}$. Let $\boldsymbol{\Phi}=\left[\mathbf{u}_{n} \otimes \mathbf{u}_{k_{1} ; \mathbf{u}_{n} \otimes \mathbf{u}_{k_{2}} ; \cdots ; \mathbf{u}_{n} \otimes \mathbf{u}_{k_{P}}}\right]$ be the $P \times L(2 M+1)$ channel model dictionary matrix of the $n$th OFDM symbol with $P$ pilots, in which $\mathbf{u}_{k_{p}}=\mathbf{u}_{k \mid k=k_{p}}$ and the pilot placement is $\mathbf{p}=\left[k_{1}, k_{2}, \ldots, k_{P}\right]$. Denote $\boldsymbol{\Phi}_{x}$ as the $P \times L$ dominant channel model whose columns are corresponding to $\mathbf{b}_{x}$. Then, the dominant channel model $\boldsymbol{\Phi}_{x}$ can be represented as

$$
\boldsymbol{\Phi}_{x}=\left[\begin{array}{ccc}
\phi\left(k_{1}, \omega_{x}\right) & \cdots & \phi\left(k_{1}, \omega_{x}+L-1\right) \\
\vdots & \ddots & \vdots \\
\phi\left(k_{P}, \omega_{x}\right) & \cdots & \phi\left(k_{P}, \omega_{x}+L-1\right)
\end{array}\right]
$$

where $\omega_{x}=L(M+x)$. Note that the columns of $\boldsymbol{\Phi}_{x}$ represents the resolvable paths and the rows represents the pilot subcarriers.

Similarly, the structure of $\boldsymbol{\Phi}=\left[\boldsymbol{\Phi}_{-M}, \ldots, \boldsymbol{\Phi}_{0}, \ldots, \boldsymbol{\Phi}_{M}\right]$ is shown in Fig. 3. In particular, $\boldsymbol{\Phi}_{M}$ with the cross lines denotes the dominant channel model with $f_{d_{\max }}$ at position $A$ corresponding to $\mathbf{b}_{M}, \boldsymbol{\Phi}_{0}$ with the slash lines denotes the one with $f_{d}=0$ at $B$, and $\boldsymbol{\Phi}_{-M}$ with the back slash lines denotes the one with $-f_{d_{\max }}$ at $C$, respectively. When the HST moves from $A$ to $C, \boldsymbol{\Phi}_{x}$ changes from $\boldsymbol{\Phi}_{M}$ to $\boldsymbol{\Phi}_{-M}$ in sequence. More specifically, as the dominant coefficients only exist in $\mathbf{b}_{x}$ corresponding to a certain Doppler shift $f_{d}$ or HST position $\alpha$, we only need to consider the ability of $\boldsymbol{\Phi}_{x}$ and estimate $\mathbf{b}_{x}$, which highly reduce the analysis and computational complexity. 


\section{B. Position-Based Channel Estimation}

Using the proposed position-based channel model earlier, we propose a channel estimation method based on the comb-type pilot. Assume that there are $P(P \leq K)$ pilots and insert at the pilot placement set $\mathbf{p}=\left[k_{1}, k_{2}, \ldots, k_{P}\right]$. The pilot placement and pilot symbols are fixed during one OFDM symbol. Since we only consider the system in one OFDM symbol, the superscripts $n$ in the remainder of this paper are omitted for compactness. Then, based on the proposed position-based channel model, (4) of the received pilot vector can be represented as follows:

$$
\begin{aligned}
\mathbf{Y}(\mathbf{p}) & =\mathbf{X}_{d}(\mathbf{p}) \mathbf{H}_{v e c}(\mathbf{p})+\mathbf{d}+\mathbf{W}(\mathbf{p}) \\
& =\mathbf{X}_{d}(\mathbf{p}) \boldsymbol{\Phi} \mathbf{b}+\mathbf{d}+\mathbf{W}(\mathbf{p}) \\
& =\mathbf{X}_{d}(\mathbf{p}) \boldsymbol{\Phi}_{x} \mathbf{b}_{x}+\mathbf{d}+\mathbf{W}(\mathbf{p})
\end{aligned}
$$

in which $\mathbf{Y}(\mathbf{p})=\left[Y\left(k_{1}\right), Y\left(k_{2}\right), \ldots, Y\left(k_{P}\right)\right]^{T}$ denotes the received pilots at the pilot placement set $\mathbf{p}, \mathbf{X}_{d}(\mathbf{p})=\operatorname{diag}\left\{\left[X\left(k_{1}\right)\right.\right.$, $\left.\left.X\left(k_{2}\right), \ldots, X\left(k_{P}\right)\right]\right\}$ denotes the transmitted signal matrix at $\mathbf{p}$, $\mathbf{H}_{v e c}(\mathbf{p})=\left[H\left(k_{1}, k_{1}\right), H\left(k_{2}, k_{2}\right), \ldots, H\left(k_{P}, k_{P}\right)\right]^{T}$ denotes the channel responses at $\mathbf{p}, \mathbf{d}=\mathbf{H}_{\mathrm{ICI}}(\mathbf{p},:) \mathbf{X}$ denotes the ICI at the pilot subcarriers, $\mathbf{H}_{\mathrm{ICI}}(\mathbf{p},:)$ denotes the rows of $\mathbf{H}_{\mathrm{ICI}}$ at $\mathbf{p}$, and $\mathbf{W}(\mathbf{p})$ denotes the AWGN at $\mathbf{p}$, respectively.

The theory of CS in [15] and [16] shows that, if $\mathbf{b}_{x}$ is $S$-sparse, which is satisfied in our system as aforementioned, then CS recovery methods can reconstruct $\mathbf{b}_{x}$ from $\mathbf{Y}(\mathbf{p})$ successfully. In this way, the task of estimating the high-mobility channel $\mathbf{H}$ in the frequency domain is converted to estimating the sparse dominant channel coefficients $\mathbf{b}_{x}$ in the delay-Doppler domain.

\section{ICI Mitigation}

In high-mobility environments, the transmitted pilots are distorted by the ICI coming from data and AWGN as represented in (16), which highly affect the channel estimation performance. In this paper, we adopt the ICI mitigation method proposed in [26]. First, the high-mobility channels can be estimated by the proposed method. As data are passed through the estimated channel, it provides an approximation of the datainduced ICI at the pilot subcarriers. Then, the estimated ICI can be subtracted at the pilot subcarriers. In this way, ICI caused by data is reduced and introduces better channel estimation performance. The process can be represented mathematically as

$$
\mathbf{Y}^{(q)}=\mathbf{Y}^{(q-1)}-\tilde{\mathbf{H}}^{(q-1)} \mathbf{z}^{(q-1)}
$$

where the superscript $q=1,2, \ldots$ denotes the iteration times. At each OFDM symbol, $\mathbf{Y}^{(0)}$ is the received signal without ICI mitigation, $\tilde{\mathbf{H}}^{(q-1)}$ is the estimated channel in the previous iteration, and $\mathbf{z}^{(q-1)}$ is the symbol constructed from the estimated data in the previous iteration with zeros at the pilot subcarriers.

In this manner, the data-induced ICI at the pilot subcarriers can be reduced and can obtain better system performance. This process can be executed with more iteration times to further reduce the ICI but limited to a certain level due to the error propagation. However, as the main topic of this paper is the positionbased channel estimator and pilot design, we do not consider the correct decision in this paper and assume that the decision feedback equalizer is error free to simplify the analysis.

\section{Coherence Optimized Pilot Design}

Here, we first review some basis of CS and then formulate the pilot design problem. A joint pilot placement and pilot symbol design algorithm is proposed and discussed. Furthermore, the practical applicability of the proposed scheme is discussed.

\section{A. CS Fundamentals}

$\mathrm{CS}$ is an innovative and revolutionary technique to reconstruct sparse signals accurately from a limited number of measurements. Given an unknown signal $\mathbf{x} \in \mathbb{C}^{m}$ and suppose $\mathbf{x}$ can be represented with a known dictionary matrix $\mathbf{D} \in \mathbb{C}^{m \times U}$ $(m<U)$ and a vector $\mathbf{a} \in \mathbb{C}^{U}$, then we have $\mathbf{x}=\mathbf{D a}$. Assume that a is $S$-sparse, i.e., $\|\mathbf{a}\|_{\ell_{0}}=S \ll U$. CS considers the following problem:

$$
\mathrm{y}=\mathbf{P x}+\eta=\mathbf{P D a}+\eta
$$

in which $\mathbf{P} \in \mathbb{C}^{p \times m}$ denotes a known measurement matrix, $\mathbf{y} \in \mathbb{C}^{p}$ denotes the observed vector, and $\boldsymbol{\eta} \in \mathbb{C}^{m}$ is the noise vector. The objective of $\mathrm{CS}$ is to reconstruct a correctly based on the knowledge of $\mathbf{y}, \mathbf{P}$, and $\mathbf{D}$. Fundamental researches [13] and [15] indicate that if $\mathbf{P D}$ satisfies the restricted isometric property (RIP) [16], then a can be reconstructed correctly with CS reconstruction methods such as the basis pursuit (BP) [27] and the orthogonal matching pursuit (OMP) [28].

To improve the CS performance, in this paper, we consider the average coherence proposed in [14]. It has been proved in [14] that the average coherence reflects the actual CS behavior rather than the mutual coherence [13] for considering the average performance. The definition is given as follows.

Definition 2 (Average Coherence [14]): For a matrix M with the $i$ th column of $\mathbf{d}_{i}$, its average coherence is defined as the average of all absolute inner products between the different normalized columns in $\mathbf{M}$ that are beyond a threshold $\delta$, where $0<\delta<1$. Put formally, we have

$$
\mu_{\delta}\{\mathbf{M}\}=\frac{\sum_{i \neq j}\left(\left|g_{i j}\right| \geq \delta\right) \cdot\left|g_{i j}\right|}{\sum_{i \neq j}\left(\left|g_{i j}\right| \geq \delta\right)}
$$

where $g_{i j}=\tilde{\mathbf{d}}_{i}^{H} \tilde{\mathbf{d}}_{j}, \tilde{\mathbf{d}}_{i}=\mathbf{d}_{i} /\left\|\mathbf{d}_{i}\right\|_{\ell_{2}}$, and the operator is defined as

$$
(x \geq y)= \begin{cases}1, & x \geq y \\ 0, & x<y\end{cases}
$$

Previous studies [13], [29] established that BP and orthogonal greedy algorithms (OGAs) (including OMP) can recover a correctly provided that the following theorem is satisfied.

Theorem 1 ([13]): For a dictionary matrix D and a measurement matrix $\mathbf{P}$, assume that $\mathbf{P D}$ satisfies RIP. If $\mathbf{y}=\mathbf{P x}=$ PDa satisfies

$$
S=\|\mathbf{a}\|_{\ell_{0}}<\frac{1}{2}\left(1+\frac{1}{\mu_{\delta}}\{\mathbf{P D}\}\right) .
$$


Then, 1) $\mathbf{a}$ is the unique sparsest representation of $\mathbf{x}$, and 2) the deviation of the reconstructed â from a by BP or OGA can be bounded by

$$
\|\hat{\mathbf{a}}-\mathbf{a}\|_{\ell_{2}}^{2} \leq \frac{\epsilon^{2}}{1-\mu_{\delta}\{\mathbf{P D}\}(2 S-1)}
$$

for some constant $\epsilon>0$.

It is easy to find that a smaller $\mu_{\delta}\{\mathbf{P D}\}$ will lead to a lower reconstruction error bound that results in a more accurate recovery of a. Furthermore, Theorem 1 implies that, if $\mathbf{P}$ is designed with a fixed $\mathbf{D}$ such that $\mu_{\delta}\{\mathbf{P D}\}$ is as small as possible, a large number of candidate signals are able to reside under the umbrella of successful CS behavior and lead to better performance.

\section{B. Problem Formulation}

As we have already known that a lower $\mu_{\delta}$ leads to a better CS performance, we are going to reduce $\mu_{\delta}\left\{\mathbf{X}_{d}(\mathbf{p}) \mathbf{\Phi}_{x}\right\}$ in our system to get better performance. In this paper, both the pilot placement and pilot symbol of the transmitted pilot matrix $\mathbf{X}_{d}(\mathbf{p})$ are considered with the dominant channel model dictionary $\boldsymbol{\Phi}_{x}$.

Let us start from the objective of minimizing $\mu_{\delta}\left\{\mathbf{X}_{d}(\mathbf{p}) \boldsymbol{\Phi}\right\}$. This pilot design problem can be formulated as follows:

$$
\min _{\left|\mathbf{X}_{d}\right|, \mathbf{p}} \mu_{\delta}\left\{\mathbf{X}_{d}(\mathbf{p}) \mathbf{\Phi}\right\}
$$

where $\left|\mathbf{X}_{d}\right|$ denotes the pilot symbols in $\mathbf{X}_{d}(\mathbf{p})$, and $\mathbf{p}$ denotes the pilot placement set. According to Definition 2, the objective function can be represented as (24), shown at the bottom of the page, where $\phi\left(k_{i}, u\right)$ is the entry of $\boldsymbol{\Phi}$ and $0 \leq u<v \leq$ $L(2 M+1)-1$.

Suppose that all pilots and data are modulated symbols, and there are $T$ pilot power levels corresponding to $T$ pilot placement subsets $\left\{\mathbf{s}_{t}\right\}_{t \in T}$. Then, we have $\bigcup_{t=1}^{T} \mathbf{s}_{t}=\mathbf{p}$, and we define the pilot power as

$$
E_{t} \triangleq\left|X\left(k_{j_{t}}\right)\right|^{2}, \quad k_{j_{t}} \in \mathbf{s}_{t}
$$

for $j_{t} \in\{1,2, \ldots, P\}$ and $t=1,2, \ldots, T$. By taking pilot power into consideration, (24) can be represented as (26), shown at the bottom of the page.

Furthermore, we consider the proposed position-based channel model. When the HST moves to a certain position $\alpha$, the high-mobility channels can be modeled by $\mathbf{b}_{x}$ corresponding to $f_{d}$. Therefore, we only need to consider the property of the columns in $\boldsymbol{\Phi}_{x}$. The columns in $\boldsymbol{\Phi}_{x}$ can be represented as $\phi(:, u)$ and $u \in[L(M+x), L(M+x)+L-1]$. Then, (26) with $\boldsymbol{\Phi}_{x}$ can be further represented as (27), shown at the bottom of the page. By taking the expression of $\boldsymbol{\Phi}_{x}$ into consideration, we have (28), shown at the bottom of the next page. Denote $z=$ $v-u$. Then, the objective function is simplified as (29), shown at the bottom of the next page. So far, the optimization variable has been simplified from a $P \times L(2 M+1)$ matrix $\mathbf{X}_{d}(\mathbf{p}) \Phi$ to a $P \times L$ matrix $\mathbf{X}_{d}(\mathbf{p}) \boldsymbol{\Phi}_{x}$, and the number of calculations has been reduced from $\left(\begin{array}{c}L(2 M+1) \\ 2\end{array}\right)$ to $(2 M+1)\left(\begin{array}{l}L \\ 2\end{array}\right)$.

$$
\mu_{\delta}\left\{\mathbf{X}_{d}(\mathbf{p}) \boldsymbol{\Phi}\right\}=\frac{\left.\sum_{u \neq v}\left(\left.\left|\sum_{k_{i} \in \mathbf{p}}\right| X\left(k_{i}\right)\right|^{2} \phi\left(k_{i}, u\right)^{H} \phi\left(k_{i}, v\right) \mid \geq \delta\right) \cdot\left|\sum_{k_{i}} \in \mathbf{p}\right| X\left(k_{i}\right)\right|^{2} \phi\left(k_{i}, u\right)^{H} \phi\left(k_{i}, v\right) \mid}{\sum_{u \neq v}\left(\left.\left|\sum_{k_{i} \in \mathbf{p}}\right| X\left(k_{i}\right)\right|^{2} \phi\left(k_{i}, u\right)^{H} \phi\left(k_{i}, v\right) \mid \geq \delta\right)}
$$

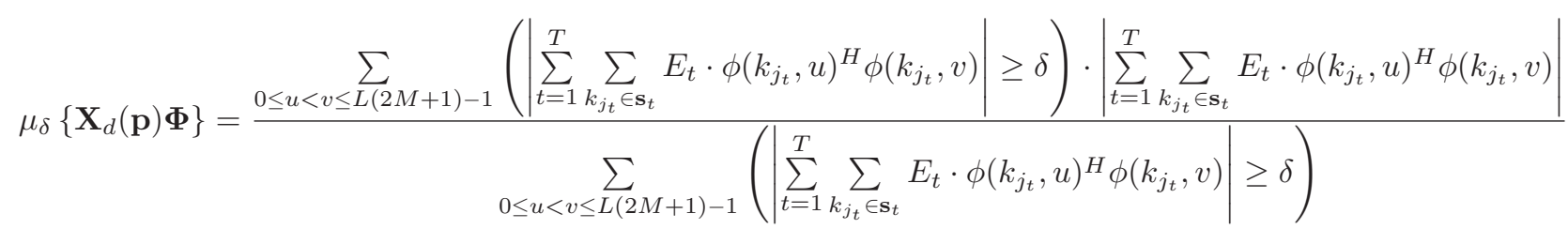

$$
\mu_{\delta}\left\{\mathbf{X}_{d}(\mathbf{p}) \mathbf{\Phi}_{x}\right\}=\frac{\sum_{u<v \text { and } u, v \in \mathbf{\Phi}_{x}}\left(\left|\sum_{t=1}^{T} \sum_{k_{j_{t}} \in \mathbf{s}_{t}} E_{t} \cdot \phi\left(k_{j_{t}}, u\right)^{H} \phi\left(k_{j_{t}}, v\right)\right| \geq \delta\right) \cdot\left|\sum_{t=1}^{T} \sum_{k_{j_{t}} \in \mathbf{s}_{t} E_{t} \cdot \phi\left(k_{j_{t}}, u\right)}^{H} \phi\left(k_{j_{t}}, v\right)\right|}{\sum_{u<v \text { and } u, v \in \mathbf{\Phi}_{x}}\left(\left|\sum_{t=1}^{T} \sum_{k_{j_{t}} \in \mathbf{s}_{t}} E_{t} \cdot \phi\left(k_{j_{t}}, u\right)^{H} \phi\left(k_{j_{t}}, v\right)\right| \geq \delta\right)}
$$


Finally, we formulate the pilot design problem as a joint optimization problem as (30) and (31), shown at the bottom of the page.

Algorithm 1: Joint Pilot Placement and Pilot Symbol Design Algorithm

Input: Initial pilot $\mathbf{X}_{0}$ with the pilot placement $\mathbf{p}_{0}$ and the pilot symbol $\mathbf{x}_{0}$.

Output: Optimal pilot $\mathbf{X}_{d}^{*}$.

1: Initialization: Set $\hat{\mathbf{X}}_{0}=\mathbf{X}_{0}$; set $M$, set Iter $=M \times P$; set pilot power $E_{1}, E_{2}, \ldots, E_{T}$; set $\mathbf{I}[0]=\mathbf{0}, I[0,0]=1$; set $\kappa=0, \iota=0$.

2: for $n=0,1, \ldots, M-1$ do

3: $\quad$ for $k=0,1, \ldots, P-1$ do

4: $\quad m \Leftarrow n \times P+k$;

$\diamond$ Generate and update:

5: $\quad$ generate $\tilde{\mathbf{p}}_{m}$ with operator $\tilde{\mathbf{p}}_{m}\left(\mathbf{p}_{m}\right)$;

6: $\quad$ if $\mu_{\delta}\left\{\mathbf{X}_{m}\left(\tilde{\mathbf{p}}_{m}\right) \boldsymbol{\Phi}_{x}\right\}<\mu_{\delta}\left\{\mathbf{X}_{m}\left(\mathbf{p}_{m}\right) \boldsymbol{\Phi}_{x}\right\}$ then

7: $\quad$ select pilot symbol power $E_{t}$ to $\min \mu_{\delta}$;

8: $\quad$ update $\mathbf{x}_{m}$ and $\mathbf{x}_{m+1} \Leftarrow \mathbf{x}_{m} ; \mathbf{p}_{m+1} \Leftarrow \tilde{\mathbf{p}}_{m}$;

9:

10:

11:

12:

13: else

select pilot symbol power $E_{t}$ to $\min \mu_{\delta}$;

update $\mathbf{x}_{m}$ and $\mathbf{x}_{m+1} \Leftarrow \mathbf{x}_{m} ; \mathbf{p}_{m+1} \Leftarrow \mathbf{p}_{m}$;

\section{end if}

update $\mathbf{X}_{m+1}$ with $\mathbf{p}_{m+1}$ and $\mathbf{x}_{m+1} ; \kappa \Leftarrow m+1$;

$\diamond$ Update state occupation probabilities:

14: $\quad \mathbf{I}[m+1] \Leftarrow \mathbf{I}[m]+\eta[m+1](\mathbf{D}[m+1]-\mathbf{I}[m])$, with $\eta[m]=1 / m$;

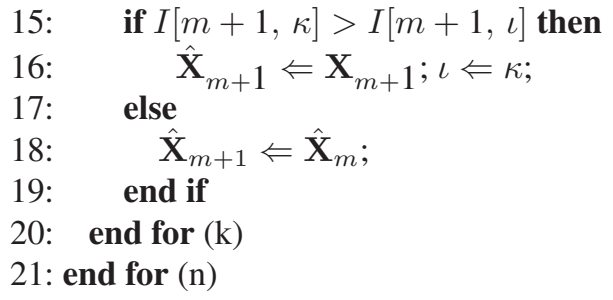

\section{Joint Pilot Placement and Pilot Symbol Design Algorithm}

An intuitive method to find the global optimal solution is to perform exhaustive search over $T^{P}\left(\begin{array}{c}K \\ P\end{array}\right)$ combinations. This method however is impractical for an OFDM system with large $K$ due to huge computational complexity. Here, we treat this optimization problem as a discrete stochastic optimization problem [30] and propose an iterative algorithm to solve it.

The key idea of our algorithm is to generate a sequence of candidate pilot matrices, where each new candidate is obtained from the previous one by taking a step in a direction toward the global optimum. The details are given in Algorithm 1. Define $\mathbf{p}_{m}, \tilde{\mathbf{p}}_{m}$, and $\hat{\mathbf{p}}_{m}$ as different pilot placement sets at the $m$ th iteration. $M$ is the number of pilot placement sets, and Iter $=M \times P$ denotes the total iteration times. At each iteration, the algorithm updates the probability vector $\mathbf{I}[m]=(I[m, 1], I[m, 2], \ldots, I[m, M P])^{T}$, which represents the state occupation probabilities of the generated pilot matrices with entries $I[m, \kappa] \in[0,1]$, and $\sum_{\kappa} I[m, \kappa]=1$. $\mathbf{D}[m] \in \mathbb{R}^{M P \times 1}$ is defined as a zero vector, except for its $m$ th entry to be 1 .

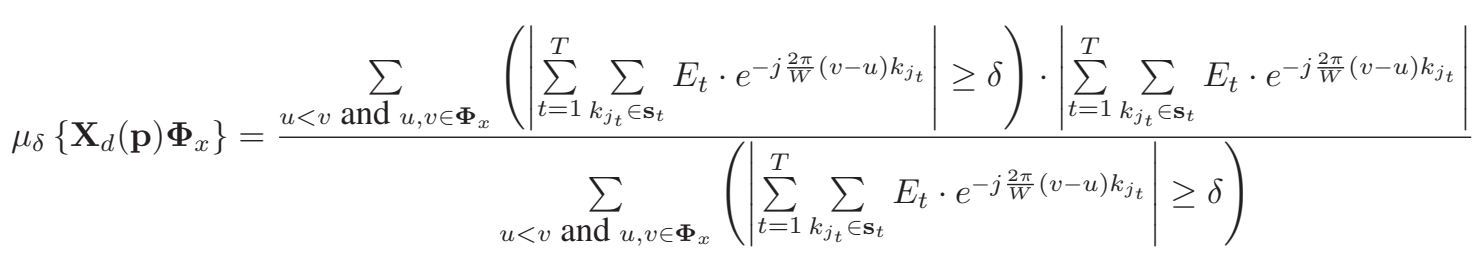

$$
\mu_{\delta}\left\{\mathbf{X}_{d}(\mathbf{p}) \boldsymbol{\Phi}_{x}\right\}=\frac{\sum_{1 \leq z \leq L-1}\left(\left|\sum_{t=1}^{T} \sum_{k_{j_{t}} \in \mathbf{s}_{t}} E_{t} \cdot e^{-j \frac{2 \pi}{W} z k_{j_{t}}}\right| \geq \delta\right) \cdot\left|\sum_{t=1}^{T} \sum_{k_{j_{t}} \in \mathbf{s}_{t}} E_{t} \cdot e^{-j \frac{2 \pi}{W} z k_{j_{t}}}\right|}{\sum_{1 \leq z \leq L-1}\left(\left|\sum_{t=1}^{T} \sum_{k_{j_{t}} \in \mathbf{s}_{t}} E_{t} \cdot e^{-j \frac{2 \pi}{W} z k_{j_{t}}}\right| \geq \delta\right)}
$$

$$
\begin{aligned}
& \mathbf{X}_{d}^{*}=\underset{\left|\mathbf{X}_{d}\right|, \mathbf{p}}{\arg \min } \mu_{\delta}\left\{\mathbf{X}_{d}(\mathbf{p}) \Phi_{x}\right\} \\
& =\underset{\mathbf{s}_{t}, E_{t}}{\arg \min } \frac{\sum_{1 \leq z \leq L-1}\left(\left|\sum_{t=1}^{T} \sum_{k_{j_{t}} \in \mathbf{s}_{t}} E_{t} \cdot e^{-j \frac{2 \pi}{W} z k_{j_{t}}}\right| \geq \delta\right) \cdot\left|\sum_{t=1}^{T} \sum_{k_{j_{t}}} \in \mathbf{s}_{t} E_{t} \cdot e^{-j \frac{2 \pi}{W} z k_{j_{t}}}\right|}{\sum_{1 \leq z \leq L-1}\left(\left|\sum_{t=1}^{T} \sum_{k_{j_{t}} \in \mathbf{s}_{t}} E_{t} \cdot e^{-j \frac{2 \pi}{W} z k_{j_{t}}}\right| \geq \delta\right)}
\end{aligned}
$$


Algorithm 1 starts with an initial pilot $\mathbf{X}_{0}$ with a random pilot placement set $\mathbf{p}_{0}$ and a random pilot symbol vector $\mathbf{x}_{0}$. In the Generate and update step, $\tilde{\mathbf{p}}_{m}$ is obtained uniformly with the operator $\tilde{\mathbf{p}}_{m}\left(\mathbf{p}_{m}\right)$. At the $m$ th iteration, the $k$ th pilot subcarrier of $\mathbf{p}_{m}$ is replaced with a random subcarrier, which is not included in $\mathbf{p}_{m}$, and then obtains $\tilde{\mathbf{p}}_{m}$. Then, we compare $\tilde{\mathbf{p}}_{m}$ with $\mathbf{p}_{m}$ and select the better one to move a step. Furthermore, we minimize the $\mu_{\delta}$ and select the best symbol power to update. In the Update state occupation probabilities step, $\mathbf{I}[m+1]$ is updated based on the previous $\mathbf{I}[m]$ with the decreasing step size $\eta[m]=1 / m . \eta[m]$ avoids the proposed algorithm moving away from a promising point unless there was strong evidence that this move will result in an improvement, which makes Algorithm 1 more progressive and conservative with increasing iterations.

\section{Global Convergence Property}

The sequence $\left\{\mathbf{X}_{m}\right\}$ generated by the proposed algorithm is a Markov chain, which in general cannot converge to a fixed point and may visit each entry infinitely often. Here, we show that the sequence $\left\{\hat{\mathbf{X}}_{m}\right\}$ surely converges to the global optimal solution $\mathbf{X}^{*}$ under certain conditions. The sufficient conditions for Algorithm 1 to converge to $\mathbf{X}^{*}$ are given as (32) and (33) [31], shown at the bottom of the page. For generated solutions $\mathbf{X}_{m} \neq \mathbf{X}^{*}$ and $\hat{\mathbf{X}}_{m} \neq \mathbf{X}^{*}$, if (32) and (33) are satisfied, then [31] proves that $\left\{\mathbf{X}_{m}\right\}$ is a homogeneous aperiodic and irreducible Markov chain in its state space. Moreover, as $\left\{\mathbf{X}_{m}\right\}$ spends much more efforts in $\mathbf{X}^{*}$ than others, $\left\{\hat{\mathbf{X}}_{m}\right\}$ surely converges to $\mathbf{X}^{*}$.

Condition (32) ensures that it is more probable for $\left\{\mathbf{X}_{m}\right\}$ to move into a state corresponding to $\mathbf{X}^{*}$ than others. Condition (33) ensures that once $\left\{\mathbf{X}_{m}\right\}$ is in a state that not corresponding to $\mathbf{X}^{*}$, it is more probable for $\left\{\mathbf{X}_{m}\right\}$ to move into a state that corresponding to $\mathbf{X}^{*}$ than others. Therefore, Algorithm 1 is a globally convergent algorithm that spends most of time at the global optimum. In addition, the property of spending more time at the global optimum than any other solution is called the attraction property of algorithms [32]. The attraction property shows that Algorithm 1 is efficient.

\section{E. Complexity Analysis}

The computational complexity is determined in terms of the number of the complex multiplications needed. The proposed algorithm consists of three steps: Initialization, Generate and update and Update state occupation probabilities. In the Initialization step, matrices and parameters are precomputed and stored in the memory; therefore, its computational complexity can be omitted. The Generate and update step computes the objective function with different pilots, which requires
$M(T+2) P^{2} L(L-1) / 2$ complex multiplications. Note that the proposed position-based channel estimator highly reduces the multiplications of this step from $M(T+2) P^{2} L(2 M+$ 1) $[L(2 M+1)-1] / 2$, particularly for a large $M$ corresponding to a high Doppler shift. Regarding the Update state occupation probabilities step, this update requires $M^{2} P^{2}$ multiplications. Therefore, Algorithm 1 requires $M(T+2) P^{2} L(L-1) / 2+$ $M^{2} P^{2}$ complex multiplications in total. In a practical system, $M, L$, and $T$ are constant parameters and much smaller than $N$. Since the pilot number $P$ is much smaller than $N$ in practice, the computational complexity of Algorithm 1 is much lower than $\mathcal{O}\left(N^{2}\right)$. In contrast, the complexity of [17] is $\mathcal{O}\left(N^{3}\right)$. Furthermore, as the needed system parameters can be estimated in advance, Algorithm 1 is an offline process; thus, its complexity can be ignored in the practical system.

\section{F. Practical Applicability}

Here, we briefly discuss the applicability of the proposed scheme in a practical HST system. As aforementioned in Section II-A, the BSs are connected with optical fibers and share the instant speed and position information of the HST, which are supported by the GPS. In a practical system, system parameters (such as $\tau_{\max }, f_{d_{\max }}$, etc.) can be collected in advance. Thus, the optimal pilots (including the pilot placement and pilot symbol) for different Doppler shifts $f_{d}$ (or HST positions $\alpha$ ) can be predesigned with Algorithm 1 by selecting corresponding $\boldsymbol{\Phi}_{x}$ and then stored into a pilot codebook, which is an offline process. The relationships between $x, f_{d}$, and $\alpha$ are given as (11) and (12), respectively. This pilot codebook is equipped at both the BS and the HST.

When the HST runs, the BS obtains the instant speed and position information of the HST from the GPS and then calculates the instant $f_{d}$. At the beginning of each OFDM symbol, the BS selects the optimal pilot from the codebook according to $f_{d}$ and transmits it to estimate the channels. This transmitted pilot is also known at the HST for checking the same codebook. Note that we assume that $f_{d}$ is constant during one OFDM symbol. Thus, the selected pilot is optimal during each OFDM symbol. In this way, the proposed scheme can be well used in current HST systems without adding too much complexity. An example of the designed pilot codebook is given in the simulation results.

\section{Simulation Results}

Here, under the high-mobility environment, we illustrate the performances of the proposed pilot design method using two typical compressed channel estimators, BP [27] and OMP [28]. The MSE and the bit error rate (BER) performances are considered versus the SNR and the HST position. In addition, the performances of the conventional least squares (LS) and

$$
\begin{aligned}
& \operatorname{Pr}\left\{\mu_{\delta}\left\{\mathbf{X}_{m}^{*} \boldsymbol{\Phi}_{x}\right\}<\mu_{\delta}\left\{\mathbf{X}_{m} \boldsymbol{\Phi}_{x}\right\}\right\}>\operatorname{Pr}\left\{\mu_{\delta}\left\{\mathbf{X}_{m} \boldsymbol{\Phi}_{x}\right\}<\mu_{\delta}\left\{\mathbf{X}_{m}^{*} \boldsymbol{\Phi}_{x}\right\}\right\} \\
& \operatorname{Pr}\left\{\mu_{\delta}\left\{\mathbf{X}_{m}^{*} \boldsymbol{\Phi}_{x}\right\}<\mu_{\delta}\left\{\hat{\mathbf{X}}_{m} \boldsymbol{\Phi}_{x}\right\}\right\}>\operatorname{Pr}\left\{\mu_{\delta}\left\{\hat{\mathbf{X}}_{m} \boldsymbol{\Phi}_{x}\right\}<\mu_{\delta}\left\{\mathbf{X}_{m}^{*} \boldsymbol{\Phi}_{x}\right\}\right\}
\end{aligned}
$$


TABLE I

HST COMMUNiCATION SySTEM PARAMETERS

\begin{tabular}{ccc}
\hline Parameters & Variables & Values \\
\hline BS power range & $R$ & $1200 \mathrm{~m}$ \\
Distance between BSs & $D s$ & $1000 \mathrm{~m}$ \\
Max distance of BS to railway & $D_{\max }$ & $1200 \mathrm{~m}$ \\
Direct distance of BS to railway & $D_{0}$ & $50 \mathrm{~m}$ \\
Carrier frequency & $f_{c}$ & $2.35 \mathrm{GHz}$ \\
Train speed & $v$ & $300-500 \mathrm{~km} / \mathrm{h}$ \\
Light speed & $c$ & $3 \times 10^{8} \mathrm{~m} / \mathrm{s}$ \\
\hline
\end{tabular}

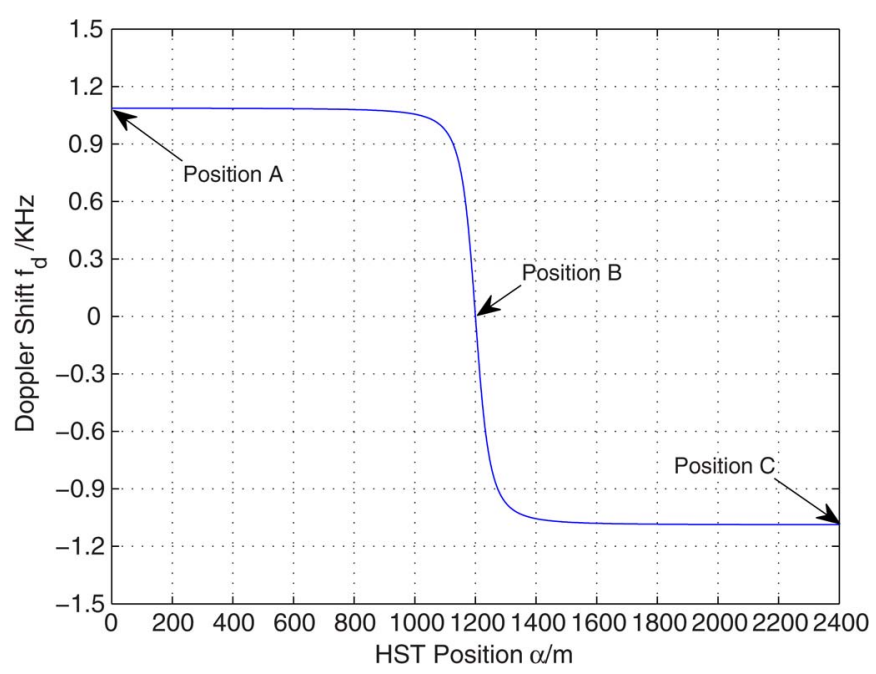

Fig. 4. Doppler shift versus HST position.

the linear MMSE (LMMSE) [9] estimators are also considered. Furthermore, we give an example of the designed pilot codebook.

Here, we consider an OFDM system in the HST communication system shown in Fig. 1. The parameters of the HST communication system are given in Table I, according to the D2a scenario of WINNER II channel model [33]. Assume that there are 512 subcarriers, and $12.5 \%$ are pilot subcarriers. The bandwidth is $5 \mathrm{MHz}$, the packet duration is $T_{d}=0.675 \mathrm{~ms}$, and the carrier frequency $f_{c}=2.35 \mathrm{GHz}$, according to [24]. Data and pilots are modulated with 16-ary quadratic amplitude modulation. The additive noise is a Gaussian and white random process. The maximum delay spread is $\tau_{\max }=5 \mu \mathrm{s}$, and the maximum Doppler shift is $f_{d_{\max }}=1.088 \mathrm{KHz}$, which means that the maximum speed of the HST is $500 \mathrm{~km} / \mathrm{h}$. The channel has $L=26$ taps; however, only six taps are nonzero, and their positions are randomly generated. The ICI mitigation is operated as mentioned in Section III-C.

\section{A. Doppler Shift Versus HST Position}

Fig. 4 shows the Doppler shift of the proposed position-based high-mobility OFDM system versus the HST position. The speed of HST is $500 \mathrm{~km} / \mathrm{h}$. In Fig. 4, the Doppler shift changes from $f_{d_{\max }}$ to $-f_{d_{\max }}$ corresponding to the HST position $\alpha$. $\alpha$ is defined as the distance between the HST and $A$, and $\alpha=0$ at $A$. We can find that HST suffers from large Doppler shift at most of the time, and $f_{d}$ changes sharply near $B$.

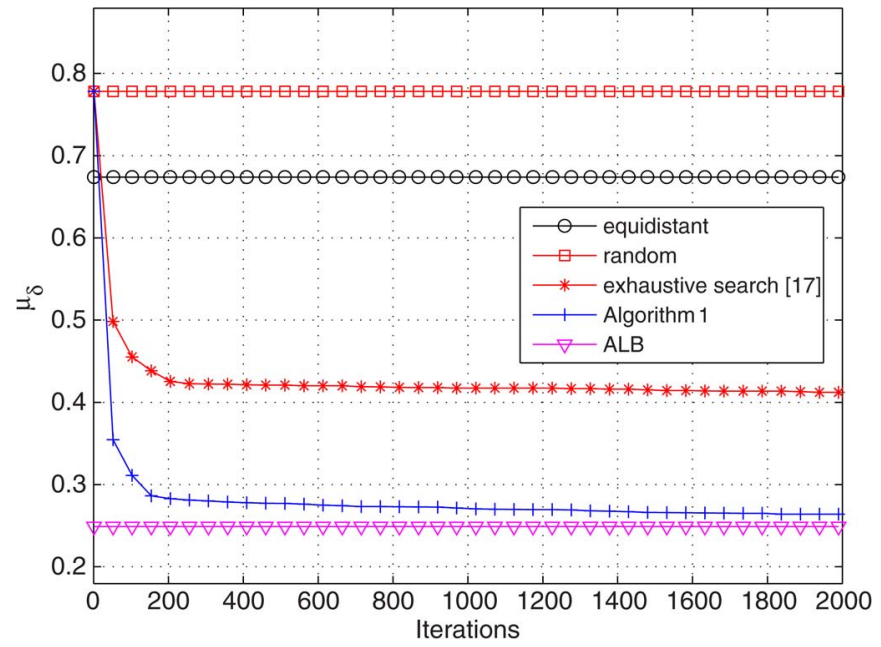

Fig. 5. Average (500 runs) of average coherence $\mu_{\delta}\left\{\mathbf{X}_{d}(\mathbf{p}) \boldsymbol{\Phi}_{x}\right\}$ with different pilot design methods in an OFDM system with $500 \mathrm{~km} / \mathrm{h}$.

\section{B. Average Coherence Versus Iterations}

In Fig. 5, we consider 500 channel realizations and gives the average coherence $\mu_{\delta}$ performance. The equidistant method is the pilot with the equidistant pilot placement and random pilot symbols, which is claimed in [10] as the optimal pilot placement to the doubly selective channels. We also plot the exhaustive search method in [17] whose main idea is to do an exhaustive search from a designed optimal pilot subset. The Algorithm 1 with Iter $=1 \times 10^{4}$ is given to show the approximate lower bound (ALB) of Algorithm 1, which means that the performance improves extremely little by increasing iterations. As can be seen, Algorithm 1 converges fast before Iter $=200$ and then converges to its ALB slowly. Note that the pilot placement iteration times is $M=(\operatorname{Iter} / P) \approx 3$ at Iter $=$ 200, which means that Algorithm 1 calculates 3 set of pilots and then gets the optimal pilot. Considering the tradeoff of the computational complexity and the estimation performance, we select Iter $=200$ in the following simulations.

\section{MSE Versus SNR}

Fig. 6 shows the comparison of the MSE performances of different estimators with different pilots with $500 \mathrm{~km} / \mathrm{h}$ at position $A$. The ICI mitigation is operated with $q=2$. The exhaustive 1 method is the method in [17] with 200 iterations, and the exhaustive 2 method denotes the same method with $2 \times 10^{4}$ iterations. The number of iterations of Algorithm 1 is set to be 200 and 2000. It can be observed that the BP channel estimators significantly improve the performance by utilizing the inherent sparsity of the high-mobility channels. As expected, Algorithm 1 has better performance than others. Furthermore, we notice that LS and LMMSE need more pilots to obtain better CSI, whereas the proposed scheme performs well and saves spectrum resources. In addition, BP with the ALB pilot is given to show the convergence tendency of Algorithm 1. It can be seen that BP-Algorithm 1 converges to BP-ALB with increasing iterations. From the curves, it is possible to observe that Algorithm 1 with Iter $=200$ is enough for the practical system. 


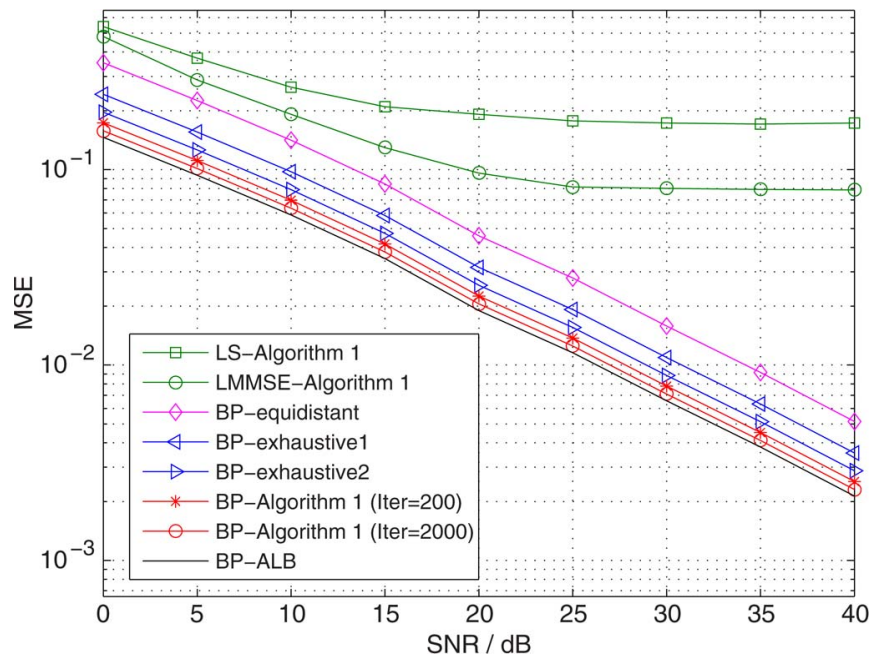

Fig. 6. MSE performances of liner estimators and BP estimators with different pilots in an OFDM system at position $A$ with $500 \mathrm{~km} / \mathrm{h}$.

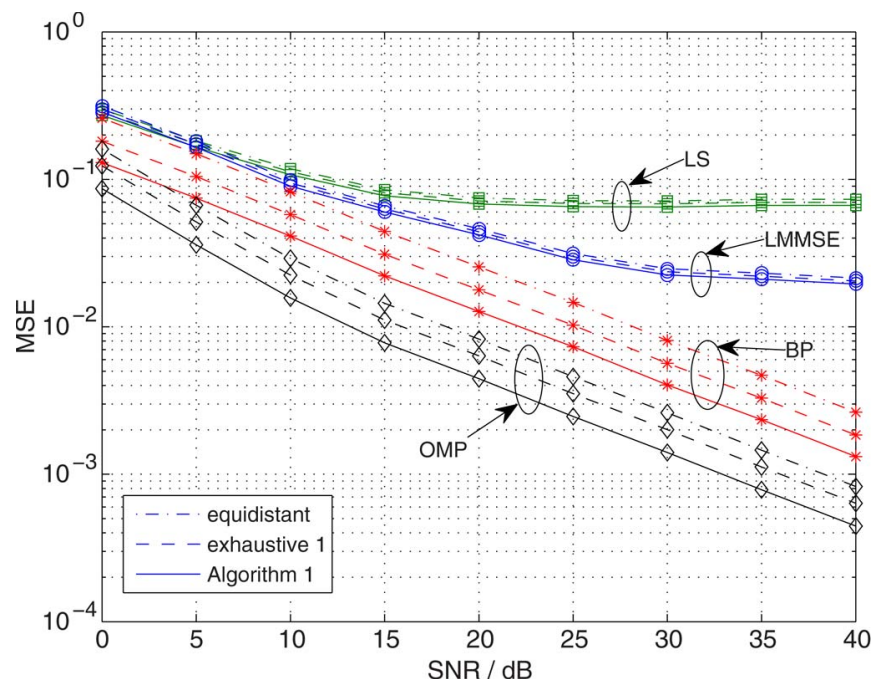

Fig. 7. MSE performances of different estimators with different pilots in an OFDM system at position $A$ with $325 \mathrm{~km} / \mathrm{h}$.

Fig. 7 depicts the comparison of the MSE performances of different estimators versus SNR at position $A$ with $325 \mathrm{~km} / \mathrm{h}$, which means $f_{d}=0.707 \mathrm{KHz}$. The ICI mitigation is operated with $q=2$, and Algorithm 1 is operated with Iter $=200$. As can be seen, with Algorithm 1, BP and OMP get better performances comparing with other pilots. On the other hand, LS and LMMSE with different pilots are also given in this figure. We notice that Algorithm 1 has little impact on linear estimators for their not utilizing the coherence of CS.

\section{BER Versus SNR}

Fig. 8 shows the BER performances versus SNR in the given high-mobility environment at position $A$ with $500 \mathrm{~km} / \mathrm{h}$. As a reference, we plot the BER performance under the perfect knowledge of CSI with Algorithm 1, which means that $\mathbf{H}$ in (1) is available at the receiver and employed with the zero-forcing equalizer. In this figure, we compare different estimators with the pilots designed by the equidistant method, the exhaustive 1 method, the exhaustive 2 method, and Algorithm $1($ Iter $=200)$.

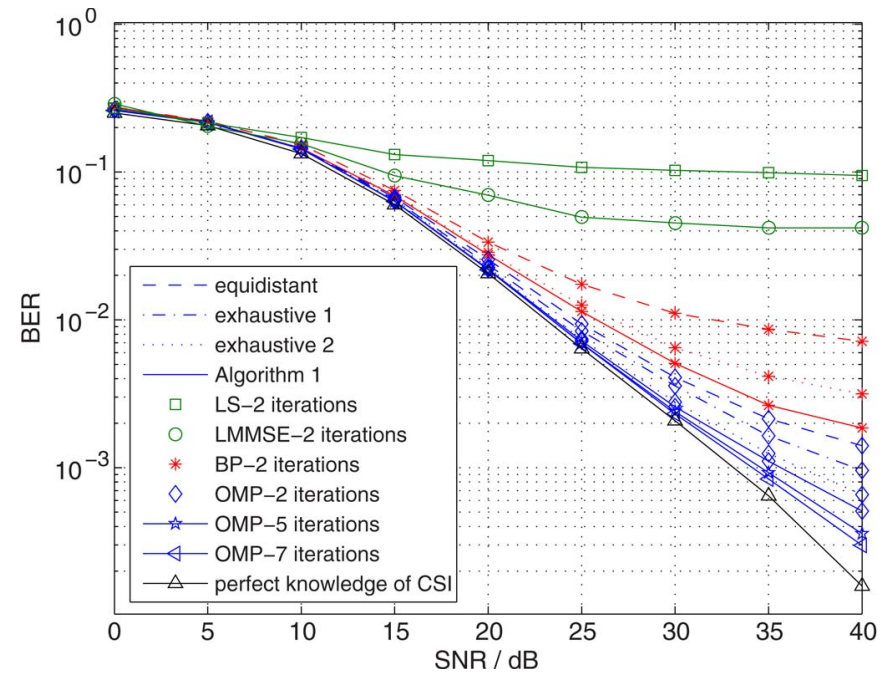

Fig. 8. BER performances of different estimators with different pilots and ICI mitigation iterations in an OFDM system at position $A$ with $500 \mathrm{~km} / \mathrm{h}$.

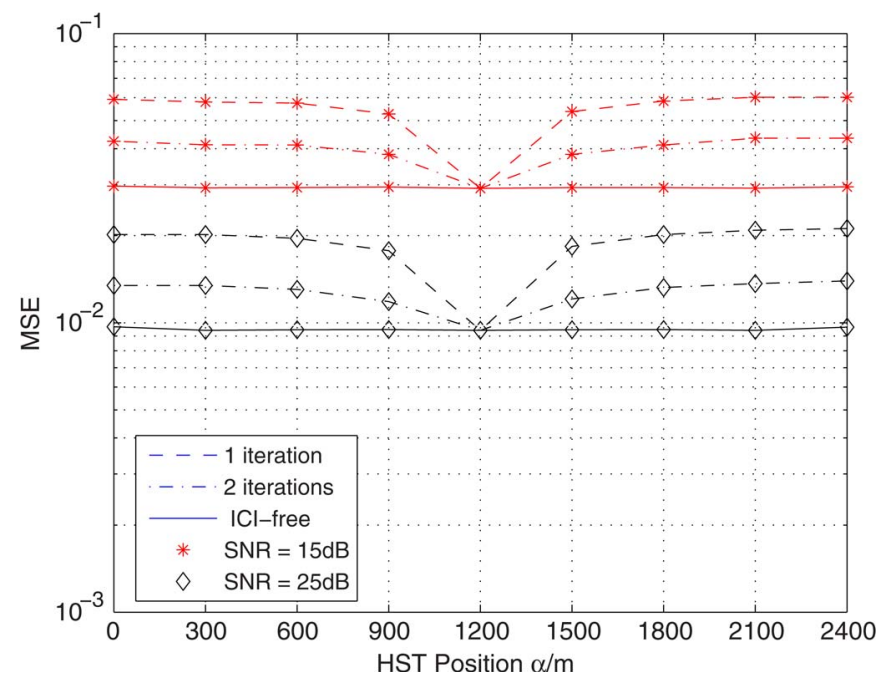

Fig. 9. MSE performances of BP estimators with Algorithm 1 versus HST positions in an OFDM system at $500 \mathrm{~km} / \mathrm{h}$.

ICI mitigation with 2,5 , and 7 iterations are considered to show its performance tendency. OMP-5 and OMP-7 are both operated with Algorithm 1. As can be seen, BP and OMP with Algorithm 1 get significant improvements and are close to the perfect knowledge of CSI, particularly in regions of low and moderate SNRs. This is mainly because, at low SNR, the noise is dominant with respect to the ICI. We also notice that the ICI mitigation gain is limited with increasing iterations due to error propagation.

\section{E. MSE Versus HST Position}

Fig. 9 presents the MSE performances of BP estimators versus the HST position at $\mathrm{SNR}=15 \mathrm{~dB}$ and $\mathrm{SNR}=25 \mathrm{~dB}$, in which the Doppler shift $f_{d}$ is corresponding to the HST position $\alpha$ shown in Fig. 4. All estimators are considered with the pilot designed by Algorithm 1 (Iter $=200)$. The train speed is fixed as $500 \mathrm{~km} / \mathrm{h}$, and $f_{d}$ changes from $f_{d_{\max }}$ to $-f_{d_{\max }}$ with the HST moving from $A$ to $C$, as shown in Fig. 4. As a reference, the performance with ICI free are also included, in which 


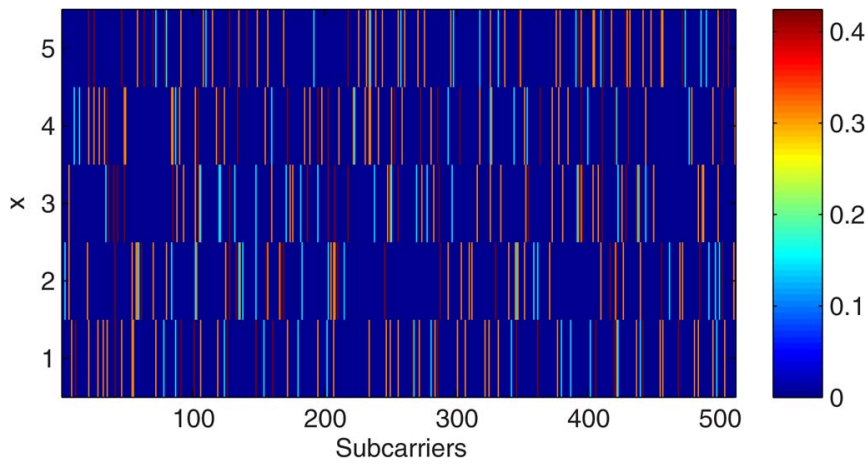

Fig. 10. A pilot codebook designed by Algorithm 1.

"ICI-free" means the data are set as zero and that pilots are free of data ICI. We notice that the MSE performances change with the HST position and obtain the best at $B$. This is reasonable because $f_{d}$ is largest at $A$ and $C$ but zero at $B$. This figure shows that the HST suffers from large Doppler shift at most of the time. However, the MSE performances improve rapidly near $B$ with decreasing Doppler shift. It can be seen that the proposed channel model well reflects the relationship between the Doppler shift and the HST position.

\section{F. Pilot Codebook for Practical Use}

Fig. 10 gives a pilot codebook designed by Algorithm 1 (Iter $=200)$ of the given system, which shows the optimal pilot sets (including the pilot placement and pilot symbol) according to the Doppler shift $f_{d}$ (or HST position $\alpha$ ). $x$ denotes the index of the pilot set, and its relationship with $f_{d}$ and $\alpha$ are given as (11) and (12), respectively. The pilot symbol power levels are presented as different colors. Furthermore, data are set to be zero for stressing the pilot placement. When the HST position changes, the optimal pilot changes according to the instant $f_{d}$. According to different $f_{d}$, the BS and RS calculate $x$ with (11) and select the optimal pilot set from the codebook. For example, $x=5$ is selected for $f_{d} \in[0.7407,1.088] \mathrm{KHz}$ (near $A$ ), and $x=1$ is selected for $f_{d} \in[-1.088,-0.7407] \mathrm{KHz}$ (near $C$ ), respectively. Meanwhile, as $f_{d}$ changes rapidly when the HST passes $B$, pilot sets $x=4,3,2$ are selected in sequence. From Fig. 4, we notice that the HST suffers from high Doppler shift at most of the time while passing through a cell. Thus, we do not need to change the pilot set frequently while the HST runs (except for the positions near $B$ ), which highly reduce the system complexity.

\section{CONCLUSION}

In this paper, we presented a new position-based compressed channel estimation method for high-mobility OFDM systems. The estimation complexity is reduced by the proposed channel model by utilizing the position information. The pilot symbol and the placement are jointly designed by the proposed algorithm to minimize the system average coherence. Simulation results demonstrate that the proposed method achieves better performances than existing channel estimation methods over high-mobility channels. Furthermore, with a predesigned pilot codebook, the proposed scheme is feasible for many current wireless OFDM communication systems.

\section{REFERENCES}

[1] P. Schniter, "Low-complexity equalization of OFDM in doubly selective channels," IEEE Trans. Signal Process., vol. 52, no. 4, pp. 1002-1011, Apr. 2004.

[2] O. B. Karimi, J. Liu, and C. Wang, "Seamless wireless connectivity for multimedia services in high speed trains," IEEE J. Sel. Areas Commun., vol. 30, no. 4, pp. 729-739, May 2012.

[3] W. U. Bajwa, A. M. Sayeed, and R. Nowak, "Sparse multipath channels: Modeling and estimation," in Proc. IEEE Digit. Signal Process. Educ. Workshop, Jan. 2009, pp. 320-325.

[4] W. U. Bajwa, J. Haupt, A. M. Sayeed, and R. Nowak, "Compressed channel sensing: A new approach to estimating sparse multipath channels," Proc. IEEE, vol. 98, no. 6, pp. 1058-1076, Jun. 2010.

[5] W. U. Bajwa, A. M. Sayeed, and R. Nowak, "Learning sparse doublyselective channels," in Proc. 46th Annu. Allerton Conf. Commun., Control Comput., Sep. 2008, pp. 575-582.

[6] S. Sung and D. Brady, "Spectral spatial equalization for OFDM in time varying frequency-selective multipath channels," in Proc. IEEE Workshop Sensor Array Multichannel Signal Process., 2000, pp. 434-438.

[7] Y. Mostofi and D. C. Cox, "ICI mitigation for pilot-aided OFDM mobile systems," IEEE Trans. Wireless Commun., vol. 4, no. 2, pp. 765-774, Mar. 2005.

[8] H. Hijazi and L. Ros, "Polynomial estimation of time-varying multipath gains with intercarrier interference mitigation in OFDM systems," IEEE Trans. Veh. Technol., vol. 58, no. 1, pp. 140-151, Jan. 2009.

[9] Z. Tang, R. C. Cannizzaro, G. Leus, and P. Banelli, "Pilot-assisted timevarying channel estimation for OFDM systems," IEEE Trans. Signal Process., vol. 55, no. 5, pp. 2226-2238, May 2007.

[10] X. Ma, G. Giannakis, and S. Ohno, "Optimal training for block transmissions over doubly-selective wireless fading channels," IEEE Trans. Signal Process., vol. 51, no. 5, pp. 1351-1366, May 2003.

[11] G. Taubock, F. Hlawatsch, D. Eiwen, and H. Rauhut, "Compressive estimation of doubly selective channels in multicarrier systems: Leakage effects and sparsity-enhancing processing," IEEE J. Sel. Topics Signal Process., vol. 4, no. 2, pp. 255-271, Apr. 2010.

[12] G. Taubock and F. Hlawatsch, "A compressed sensing technique for OFDM channel estimation in mobile environments: Exploiting channel sparsity for reducing pilots," in Proc. IEEE ICASSP, Mar. 2008, pp. 2885-2888.

[13] D. L. Donoho, M. Elad, and V. N. Temlyakov, "Stable recovery of sparse overcomplete representations in the presence of noise," IEEE Trans. Inf. Theory, vol. 52, no. 1, pp. 6-18, Jan. 2006.

[14] M. Elad, "Optimized projections for compressed sensing," IEEE Trans. Signal Process., vol. 55, no. 12, pp. 5695-5702, Dec. 2007.

[15] E. J. Candes, J. Romberg, and T. Tao, "Robust uncertainty principles: Exact signal reconstruction from highly incomplete frequency information," IEEE Trans. Inf. Theory, vol. 52, no. 2, pp. 489-509, Feb. 2006.

[16] E. J. Candes and M. B. Wakin, "An introduction to compressive sampling," IEEE Signal Process. Mag., vol. 25, no. 2, pp. 21-30, Mar. 2008.

[17] X. He and R. Song, "Pilot pattern optimization for compressed sensing based sparse channel estimation in OFDM systems," in Proc. Int. Conf. WCSP, Oct. 2010, pp. 1-5.

[18] N. Jing, W. Bi, and L. Wang, "Deterministic pilot design for MIMO OFDM system based on compressed sensing," in Proc. ICCT, Nov. 2012, pp. 897-903.

[19] D. Wang and X. Hou, "Compressed MIMO channel estimation and efficient pilot pattern over Doppler sparse environment," in Proc. Int. Conf. WCSP, Nov. 2011, pp. 1-5.

[20] C. Qi and L. Wu, "Optimized pilot placement for sparse channel estimation in OFDM systems," IEEE Signal Process. Lett., vol. 18, no. 12, pp. 749-752, Dec. 2011.

[21] C. Qi and L. Wu, "A study of deterministic pilot allocation for sparse channel estimation in OFDM systems," IEEE Commun. Lett., vol. 16, no. 5, pp. 742-744, May 2012.

[22] X. Ren, W. Chen, and Z. Wang, "Low coherence compressed channel estimation for high mobility MIMO OFDM systems," in Proc. IEEE GLOBECOM, Dec. 2013, pp. 3389-3393.

[23] X. Xiao, B. Zheng, and C. Wang, "Compressed channel estimation based on optimized measurement matrix," in Proc. WCSP, Nov. 2011, pp. 1-5.

[24] L. Liu et al., "Position-based modeling for wireless channel on highspeed railway under a viaduct at $2.35 \mathrm{GHz}$," IEEE J. Sel. Areas Commun., vol. 30, no. 4, pp. 834-845, May 2012.

[25] R. D. Pascoe and T. N. Eichorn, "What is communication-based train control?" IEEE Veh. Technol. Mag., vol. 4, no. 4, pp. 16-21, Dec. 2009.

[26] H. Hijazi and L. Ros, "Joint data QR-detection and kalman estimation for OFDM time-varying rayleigh channel complex gains," IEEE Trans. Commun., vol. 58, no. 1, pp. 170-178, Jan. 2010. 
[27] S. S. Chen, D. L. Donoho, and M. A. Saunders, "Atomic decomposition by basis pursuit," Soc. Ind. Appl. Math. Rev., vol. 43, no. 1, pp. 129-159, 2001.

[28] Y. C. Pati, R. Rezaiifar, and P. S. Krishnaprasad, "Orthogonal matching pursuit: Recursive function approximation with applications to wavelet decomposition," in Proc. 27th Annu. Asilomar Conf. Signals, Syst. Comput., Nov. 1993, vol. 1, pp. 40-44.

[29] J. A. Tropp, "Greed is good: Algorithmic results for sparse approximation," IEEE Trans. Inf. Theory, vol. 50, no. 10, pp. 2231-2242, Oct. 2004.

[30] I. Berenguer, X. Wang, and V. Krishnamurthy, "Adaptive MIMO antenna selection via discrete stochastic optimization," IEEE Trans. Signal Process., vol. 53, no. 11, pp. 4315-4329, Nov. 2005.

[31] S. Andradottir, "A global search method for discrete stochastic optimization," Soc. Ind. Appl. Math. J. Optim., vol. 6, no. 2, pp. 513-530, 1996.

[32] S. Andradottir, "Accelerating the convergence of random search methods for discrete stochastic optimization," ACM Trans. Modeling Comput. Simul., vol. 9, no. 4, pp. 349-380, Oct. 1999.

[33] K. Pekka et al., "WINNER II Channel Models. IST-4-027756," WirelessWorld-Initiative-New-Radio (WINNER II), Munich, Germany, Tech. Rep. D1.1.2 v1.1, Sep. 2007.

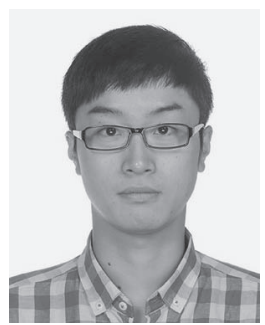

Xiang Ren received the B.S. degree in electronic engineering from Wuhan University, Wuhan, China, in 2011. He is currently working toward the Ph.D. degree with the Network Coding and Transmission Laboratory, Department of Electronic Engineering, Shanghai Jiao Tong University, Shanghai, China.

His current research interests include channel estimation, high mobility channels, and multipleinput-multiple-input orthogonal-frequency-divisionmultiplexing systems.

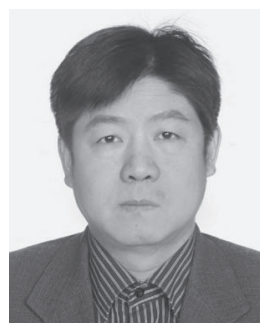

Wen Chen (M'03-SM'11) received the B.S. and M.S. degrees from Wuhan University, Wuhan, China, in 1990 and 1993, respectively, and the Ph.D. degree from The University of Electro-Communications, Tokyo, Japan, in 1999.

From 1999 to 2001, he was a Researcher with the Japan Society for the Promotion of Sciences. In 2001, he joined the University of Alberta, Edmonton, $\mathrm{AB}$, Canada, starting as a Postdoctoral Fellow with the Information Research Laboratory and continuing as a Research Associate with the Department of Electrical and Computer Engineering. Since 2006, he has been a Full Professor with the Department of Electronic Engineering, Shanghai Jiao Tong University, Shanghai, China, where he is also the Director of the Institute for Signal Processing and Systems. Since 2014, he has also been the Dean of Electronic Engineering and Automation with Guilin University of Electronic Technology, Guilin, China. He is the author of 54 papers in IEEE JOURNALS AND TRANSACTIONS and more than 100 papers in IEEE Conference Publications. His research interests include network coding, cooperative communications, cognitive radio, and multiple-input-multiple-output orthogonal-frequency-divisionmultiplexing systems.

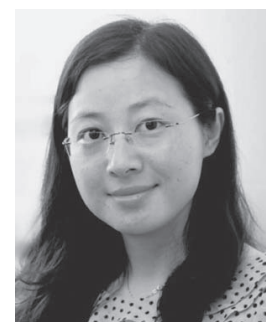

Meixia Tao (S'00-M'04-SM'10) received the B.S. degree in electronic engineering from Fudan University, Shanghai, China, in 1999 and the Ph.D. degree in electrical and electronic engineering from Hong Kong University of Science and Technology, Clear Water Bay, Hong Kong, in 2003.

From 2003 to 2004, she was a member of the Professional Staff with Hong Kong Applied Science and Technology Research Institute. From 2004 to 2007, she was a Teaching Fellow and then an Assistant Professor with the Department of Electrical and Computer Engineering, National University of Singapore, Singapore. She is currently a Professor with the Department of Electronic Engineering, Shanghai Jiao Tong University, Shanghai, China. Her current research interests include cooperative communications, wireless resource allocation, multipleinput-multiple-output techniques, and physical-layer security.

Dr. Tao is an Editor for the IEEE TRANSACTIONS ON COMMUNICATIONS and the IEEE WirELESS COMMUNiCATIONS LETTERS. She was on the Editorial Board of the IEEE TRANSACTIONS ON WIRELESS COMMUNICATIONS from 2007 to 2011 and the IEEE COMMUNICATIONS LETTERS from 2009 to 2012. She also served as a Guest Editor for IEEE COMMUNICATIONS MAGAZINE with a feature topic on Long-Term Evolution-Advanced and fourthgeneration Wireless Communications in 2012 and for the EURASIP Journal on Wireless Communications and Networking with a special issue on physicallayer network coding for wireless cooperative networks in 2010. She received the IEEE Communication Society Asia-Pacific Outstanding Young Researcher Award in 2009, the International Conference on Wireless Communications and Signal Processing Best Paper Award in 2012, and the IEEE Heinrich Hertz Award for Best Communications Letters in 2013. 